\title{
Adherence and virologic suppression during the first 24 weeks on antiretroviral therapy among women in Johannesburg, South Africa - a prospective cohort study
}

Ziad El-Khatib ${ }^{1,2}$, Anna Mia Ekstrom ${ }^{1}$, Ashraf Coovadia ${ }^{3}$, Elaine J Abrams ${ }^{4}$, Max Petzold ${ }^{5 *}$, David Katzenstein ${ }^{6}$, Lynn Morris ${ }^{2}$, Louise Kuhn

\begin{abstract}
Background: Adherence is a necessary part of successful antiretroviral treatment (ART). We assessed risk factors for incomplete adherence among a cohort of HIV-infected women initiating ART and examined associations between adherence and virologic response to ART.

Methods: A secondary data analysis was conducted on a cohort of 154 women initiating non-nucleoside reverse transcriptase inhibitor (NNRTI)-based ART at a single site in Johannesburg, South Africa. Ninety women had been enrolled in a prevention of mother-to-child transmission (PMTCT) program and were exposed to single-dose nevirapine (sdNVP) $>18$ months earlier. Women were interviewed pre-treatment and clinical, virologic and adherence data were collected during follow-up to 24 weeks. Incomplete adherence to ART was defined as returning $>5 \%$ of medications, estimated by pill counts at scheduled visits. Multivariable logistic regression analysis and unadjusted odds ratio $(95 \% \mathrm{Cl})$ were performed, using STATA/SE (ver 10.1).

Results: About half of the women (53\%) were $<30$ years of age, $63 \%$ had $<11$ years of schooling, $69 \%$ were unemployed and 37\% lived in a shack. Seven percent of women had a viral load $>400$ copies $/ \mathrm{ml}$ at 24 weeks and $37 \%$ had incomplete adherence at one or more visits. Incomplete adherence was associated with less education ( $p=$ $0.01)$ and lack of financial support from a partner $(p=0.02)$ after adjustment for confounders. Only when adherence levels dropped below $80 \%$ was there a significant association with viremia in the group overall $(p=0.02)$ although adherence $<95 \%$ was associated with viremia in the sdNVP-exposed group $(p=0.03)$. The main reasons for incomplete adherence were being away from home, busy with other things and forgetting to take their medication.

Conclusion: Virologic response to NNRTI-treatment in the cohort was excellent. However, women who received sdNVP were at greater risk of virologic failure when adherence was $<95 \%$. Women exposed to sdNVP, and those with less education and less social support may benefit from additional adherence counseling to ensure the longterm success of ART. More than $80 \%$ adherence may be sufficient to maintain virologic suppression on NNRTIbased regimens in the short-term, however complete adherence should be encouraged.
\end{abstract}

\section{Background}

National and international organizations have committed to support antiretroviral treatment (ART) programs in sub-Saharan Africa (SSA) [1,2]. More than $60 \%$ of HIV-infected adults in SSA are women, who are

\footnotetext{
* Correspondence: max@nhv.se

${ }^{5}$ Nordic School of Public Health (NHV), Gothenburg, Sweden

Full list of author information is available at the end of the article
}

disproportionately affected by the HIV-1 epidemic for both biological and socio-cultural reasons $[1,3,4]$. Although women are more likely to seek healthcare and initiate ART earlier than men, $[4,5]$ they may be more likely to show incomplete adherence and discontinue ART during the first year on therapy [6]. Women's adherence to ART may be compromised by child-care responsibilities and dependency ratios, $[7,8]$ economic

\section{Biomed Central}


pressures and lack of partner support [8,9]. Incomplete adherence to ART increases women's risk of virologic failure and subsequent clinical progression.

The association between adherence and virologic outcome is complex [10]. Earlier, unboosted protease inhibitor-based ART regimens required more than 95\% adherence to ensure virologic suppression [11]. With today's non-nucleoside reverse transcriptase inhibitor (NNRTI)- and boosted protease inhibitor-based regimens a moderate adherence level (70-90\%) may be adequate to achieve virologic suppression [12-14]. Bangsberg and coworkers [15] found, using a medical electronic assessment (MEMS), that $>75 \%$ adherence may be sufficient to achieve virologic suppression among men receiving an NNRTI-based regimen in San Francisco. However, for optimal outcomes and to avoid disease progression [16-19], maximum level of adherence (100\%) should always be the patient's goal and the provider's recommendations [12-14]. An additional factor for women that may jeopardize ART success is prior receipt of singledose nevirapine (sdNVP) for prevention of mother-tochild transmission which can select drug resistance and jeopardize the efficacy of ART [20-23]. Little is known about the association between adherence level and virologic suppression among women exposed to sdNVP.

In this study we examined socio-economic characteristics associated with incomplete adherence, assessed through pharmacy pill counts, and HIV RNA quantified in plasma among women in a research cohort, from ART initiation through 24 weeks of treatment in Johannesburg, South Africa. The aim was to identify characteristics of women at higher risk of incomplete adherence to better target interventions to enhance adherence and sustain long-term ART success.

\section{Methods}

\section{Setting and population}

HIV-infected women were recruited into an earlier study in Johannesburg, South Africa, between July 2004 and May 2006, to assess whether previous sdNVP impacted the efficacy of ART [23]. Women who had received sdNVP as prophylaxis in a prior pregnancy 18 to 36 months earlier and women who had a pregnancy in the same interval but who did not receive sdNVP were eligible for enrollment. Other inclusion criteria included ART-naïve and (i) presenting with CD4+ cell counts $<200$ cells $/ \mathrm{mm}^{3}$, (ii) CD $4+$ cell counts $<350$ cells $/ \mathrm{mm}^{3}$ and World Health Organization (WHO) stage III, or (iii) WHO stage IV disease. Exclusion criteria were (i) acute hepatitis, (ii) elevated liver function test values or (iii) a history of nevirapine toxicity [23]. Women were initiated onto therapy with nevirapine, stavudine and lamivudine unless co-treatment for tuberculosis was required in which case efavirenz was substituted for nevirapine. The primary results of this study have been previously reported [23]. All women provided written informed consent and the study was approved by the Institutional Review Boards of Columbia University, USA and the University of the Witwatersrand, South Africa.

\section{Study procedures}

A baseline questionnaire was administered at enrolment prior to treatment initiation including questions on education level, socio-economic indicators, household members, previous pregnancies, previous live births and number of living children, marital status, quality of life (QOL) [24-26] and exposure to sdNVP. HIV RNA viral load (VL) and CD4+ cell counts were measured pretreatment and WHO clinical stage was determined. Women attended the clinic monthly to 24 weeks and at each visit VL was measured and an adherence assessment was done by the study pharmacist. At week 24, another questionnaire was administered monitoring life events and QOL since ART initiation. CD4+ cell counts and VL $\leq 400$ or $>400$ copies $/ \mathrm{ml}$ were the primary treatment outcomes.

\section{Adherence and virologic failure assessment}

Pill counts [27] were performed by the study pharmacist, who counted the number of remaining pills for each drug separately at each drug refill visit. Refills were scheduled at weeks $2,4,8,12,16,20$ and 24 from the start of treatment. Pill count-based adherence was assessed by calculating the average combined pill count for the three drugs at each visit, using the formula [Adherence $=($ Number of pills dispensed - Number of pills returned $) \times 100) /($ Number of pills prescribed daily $\times$ Number of days between pharmacy visits)].

In our study, the adherence level of women was assessed at the seven time points. Patients were defined as incompletely adherent, if they ever had an average pill count of returning back $>5 \%$ of their prescribed pills to the pharmacy, indicating an adherence level of $<95 \%$ adherence at any visit. We also performed additional analyses of levels of adherence indicated by the percentage of returned pills from $0 \%$, to $>5 \%,>10 \%,>20 \%$ and $>30 \%$ on the outcome of $\mathrm{VL}>400$ copies $/ \mathrm{ml}$ at week 24 . This analysis was done first among all women and then among women exposed vs. not exposed to sdNVP. Patients were asked a battery of questions to assess QOL, where the sum of questions varied from 12 (very good QOL) to 55 (the poorest level of QOL). These responses were categorized as 1) 12-23 (good), 2) 24-35 (intermediate) or 3) 36-55 (poor) to analyze the relationship between QOL, adherence and virologic response.

Viral load was measured using the Roche Amplicor assay (Roche Diagnostics, version 1.5) using the standard 
assay (lower limit of detection, 400 copies $/ \mathrm{ml}$ ) before commencement of treatment and the ultrasensitive assay (lower limit of detection, 50 copies $/ \mathrm{ml}$ ) after commencement of treatment. CD4+ cell count was measured by flow cytometry [23]. Viral load was assessed pre-ART initiation and every four weeks until week 24 and VL $>400$ copies/ml was defined as virologic failure. $\mathrm{CD} 4+$ cell count was assessed pre-ART initiation and every 12 weeks [23].

\section{Adherence support}

Special attention was given to adherence counseling. A patient contract was drawn up in which the participant agreed with the clinical staff to adhere as closely as possible to medication guidelines. A booklet including HIV and treatment information considered necessary for adequate adherence was developed and participants were evaluated for their understanding of this information prior to starting therapy. Pill-boxes and diary cards designed to suit the regimen were given to all participants. Each post-treatment visit included a pharmacist's review of dosing and adherence, a pill-count of returned drugs, and all members of the clinical team offered support around adherence throughout the study [23].

\section{Data analysis}

Characteristics of the participants (including demographics, socio-economic and pre-treatment clinical factors) were compiled. Associations between incomplete adherence, virologic failure and these other characteristics were assessed using unadjusted odds ratios (OR) with 95\% confidence intervals $(\mathrm{CI})$ and p-values. Backward-selection multivariable logistic regression analysis was performed including factors with $\mathrm{p}$-values $\leq 0.10$ and then repeated for those variables yielding $\mathrm{p}$-values $\leq 0.05$. Associations between incomplete adherence at different thresholds and virologic failure were assessed using unadjusted odds ratios with $95 \%$ CI. The statistical analysis was performed using STATA/SE (ver 10.1).

\section{Results}

\section{Characteristics of women pre-ART initiation}

A total of 154 women participated in this study. Of these, 147 (95\%) had pill count assessment data for at least five out of seven visits during the study period (i.e. the first 24 weeks on ART); data on seven patients (5\%) were missing for more than five visits and these were excluded from the final analysis. The socio-economic and clinical characteristics of these seven women, did not differ from the women included in the analysis (data not shown). Most of the 147 women were born in South Africa (88\%), 53\% were below 30 years of age, and $63 \%$ had less than 11 years of schooling. In terms of income, $69 \%$ were unemployed and one third (37\%) lived in an informal dwelling (shack). Most (83\%) women reported being single, $75 \%$ had been pregnant at least twice and $40 \%$ had at least two living children. When we asked about financial support from a partner or a husband, less than half of single women (38\%) and a majority of married women $(84 \%)$ reported receipt of support. The majority had more than four of the pre-specified household assets: indoor tap water (50\%), toilet $(42 \%)$, electricity $(84 \%)$, a refrigerator $(66 \%)$, radio $(82 \%)$, TV $(82 \%)$ or a landline telephone (11\%), defining higher socioeconomic status. Seventy percent had disclosed their HIV status to someone at home and $85 \%$ had a treatment buddy. Ninety women had been exposed to sdNVP in their previous pregnancy. The median levels of VL and CD4 cell count pre-ART initiation were 110,000 copies/ml (range 760 - >750,000) and 166 cells/ $\mu \mathrm{l}$ (range 2 - 430) respectively. Less than a quarter (23\%) scored poorly on QOL assessments at baseline.

\section{Risk factors for incomplete adherence}

Fifty-five patients (37\%) demonstrated incomplete adherence, as defined by ever returning $>5 \%$ of their pills at any visit through 24 weeks. Univariable analysis examining preART factors associated with incomplete adherence is shown in Table 1. In multivariable analysis, two factors remained significantly associated with incomplete adherence, namely, education level $\leq$ grade $11(\mathrm{OR}=2.6$; 95\% CI 1.2-5.6; $\mathrm{p}=0.01)$ and not receiving financial support from a partner or a husband $(\mathrm{OR}=2.3 ; 95 \%$ CI 1.1-4.8; $\mathrm{p}=0.02)$. Women who had received sdNVP as prophylaxis in a prior pregnancy were less likely to demonstrate incomplete adherence $(\mathrm{OR}=0.5 ; 95 \% \mathrm{CI} 0.2-1.0 ; \mathrm{p}=0.05)$ but this association did not persist after adjustment for confounders.

Characteristics reported at 24 weeks were also investigated as potential correlates of incomplete adherence (Table 2). The remaining risk factors associated with a trend towards incomplete adherence with p-values ranging from 0.06 to 0.10 were: birth outside South Africa, living in informal housing, not having a water source inside the home, low socio-economic status, and reporting a divorce or separation during first 24 weeks on ART.

\section{Risk factors for viral load $>\mathbf{4 0 0}$ copies $/ \mathrm{ml}$ at week $\mathbf{2 4}$}

Eleven patients $(7 \%)$ had VL $>400$ copies $/ \mathrm{ml}$ at 24 weeks, six of whom (55\%) were considered to be incompletely adherent during the study period. As shown in Table 3 there were trends between the viral outcome and incomplete adherence during the first 24 weeks on therapy $(\mathrm{OR}=2.1 ; 95 \%$ CI 0.5-9.3; $\mathrm{p}=0.33)$, an education level below or equal to grade $11(\mathrm{OR}=2.9 ; 95 \% \mathrm{CI} 0.6-28.0$; $\mathrm{p}=0.21)$, living in an informal settlement $(\mathrm{OR}=2.2 ; 95 \%$ CI 0.5-9.6; $\mathrm{p}=0.21$ ) and a death in the family during the first 24 weeks on ART $(\mathrm{OR}=3.3$; 95\% CI 0.7-14.0; $\mathrm{p}=$ $0.06)$. As has been reported previously, there was no 
Table 1 Pre-antiretroviral therapy (ART) characteristics and incomplete adherence over first 24 weeks of therapy (ever with pill count $<95 \%$ past 24 weeks)

$\begin{array}{lll}\begin{array}{l}\text { Number (\%) of those with } \\ \text { complete adherence }(\mathbf{n = 9 2 )}\end{array} & \begin{array}{l}\text { Number (\%) of those with } \\ \text { incomplete adherence } \\ (\mathbf{n = 5 5 )}\end{array} & \begin{array}{l}\text { Univariable } \\ \text { odds ratio } \\ \mathbf{9}=5 \% \mathrm{Cl}) ; \\ \mathbf{p} \text {-value }\end{array} \\ 8(9 \%) & 10(18 \%) & 1 \\ 83(91 \%) & 45(82 \%) & 0.4(0.2-1.2) ; \\ & & 0.10\end{array}$

Age (years)

$\begin{array}{llll}<30(78 ; 53 \%) & 50(54 \%) & 28(51 \%) & 1 \\ \geq 30(69 ; 47 \%) & 42(46 \%) & 27(49 \%) & 1.2(0.6-2.2) ; \\ & & & 0.69\end{array}$

Education level

\begin{tabular}{llll}
$>$ grade $11(54 ; 37 \%)$ & $41(45 \%)$ & $13(24 \%)$ & $\mathbf{1}$ \\
$\leq$ grade $11(91 ; 63 \%)$ & $50(55 \%)$ & $41(76 \%)$ & $\mathbf{2 . 6 ( 1 . 2 - 5 . 6 ) ;}$ \\
\hline
\end{tabular}

Marital status

Single/divorced/widow (122; 83\%) $74(80 \%)$

Married/partner $(25 ; 17 \%)$

Socio-economic status (SES) Employed

$\begin{array}{ll}\text { No }(101 ; 69 \%) & 64(70 \%) \\ \text { Yes }(46 ; 31 \%) & 28(30 \%)\end{array}$

Type of housing

House/flat/rented room (93; 63\%) $63(68 \%)$

Shack/outbuilding $(54 ; 37 \%)$

$29(32 \%)$

Number of children living at home
$0-1$ child $(89 ; 61 \%)$
$57(62 \%)$
$\geq 2$ children $(58 ; 39 \%)$
$35(38 \%)$

$0.6(0.2-1.5)$

Lack of financial support from partner/husband

Support $(67 ; 45 \%)$

$43(47 \%)$

$37(67 \%)$

$18(33 \%)$

$1.1(0.5-2.3)$

0.77

Lack of support $(80 ; 55 \%)$

Water source inside home
No $(74 ; 50 \%)$
$41(45 \%)$
Yes $(73 ; 50 \%)$
$51(55 \%)$

$33(60 \%)$

$22(40 \%)$

$1.8(0.9-3.6)$

0.09

$51(55 \%)$

41 (45\%)

35 (64\%)

$20(36 \%)$

Yes $(61.42 \%)$

Electricity at home

$$
\text { No }(23 ; 16 \%)
$$

Yes $(124 ; 84 \%)$

$13(14 \%)$

$10(18 \%)$

45 (82\%)

27 (29\%)

$65(71 \%)$
$23(42 \%)$

$32(58 \%)$
1

$1.2(0.6-2.3)$

0.65

1

2.3 (1.1-4.8);

0.02

1

$0.5(0.3-1.1)$; 0.07

$0.7(0.4-1.4)$; 0.33

1 0.7 (0.3-1.8); 0.51

1

$0.6(0.3-1.2) ;$ 0.12 
Table 1 Pre-antiretroviral therapy (ART) characteristics and incomplete adherence over first 24 weeks of therapy (ever with pill count $<95 \%$ past 24 weeks) (Continued)

Radio at home

$$
\begin{aligned}
& \text { No }(28 ; 19 \%) \\
& \text { Yes }(119 ; 81 \%)
\end{aligned}
$$

TV at home

$$
\text { No }(27 ; 18 \%)
$$$$
\text { Yes }(120 ; 82 \%)
$$

Landline telephone
No $(131 ; 89 \%)$
Yes $(16 ; 11 \%)$
$82(89 \%)$
$10(11 \%)$

$12(13 \%)$

$80(87 \%)$

$13(24 \%)$

$42(76 \%)$

$0.6(0.3-1.5)$ 0.28

Pre-treatment clinical characteristics Exposed to sdNVP

$$
\text { No }(57 ; 39 \%)
$$

Yes $(90 ; 61 \%)$

VL level, pre-ART (copies/ml)

Median (range)

CD4 level (cells/ $\mu \mathrm{l})$

Median (range)

Felt too sick to go to work or to do the daily activities, during the last 30 days
No $(104 ; 71 \%)$
Yes $(43 ; 29 \%)$
$64(70 \%)$
$28(30 \%)$

$62(67 \%)$

127,500

$(760->750,000)$

$155(7-430)$

Traditional medicine/herbs in the last 3 months

$\begin{array}{ll}\text { No }(126 ; 87 \%) & 79(86 \%) \\ \text { Yes }(19 ; 13 \%) & 13(14 \%)\end{array}$

Quality of life

Good (score 12-23) (38; 26\%)

Intermediate (score $24-35)(75 ; 51 \%)$

$27(29 \%)$

$43(47 \%)$

$22(24 \%)$

Poor (score 36-55) (34; 23\%)

$27(29 \%)$

$65(71 \%)$

Yes $(102 ; 70 \%)$

Treatment buddy
No $(22 ; 15 \%)$
Yes $(125 ; 85 \%)$
$11(12 \%)$
$81(88 \%)$

$29(60 \%)$

$19(40 \%)$

No $(46 ; 57 \%)$

Another child at home living with HIV
No $(12 ; 12 \%)$
$9(15 \%)$
Yes $(87 ; 88 \%)$

$15(27 \%)$

$40(73 \%)$

$49(89 \%)$

$6(11 \%)$

$40(73 \%)$

$15(27 \%)$

$27(49 \%)$

$28(51 \%)$

$(6,600->750,000)$

$189(2-324)$

1

$0.4(0.2-1.0)$

0.03

$1.0(0.3-2.9)$

0.99

$0.5(0.2-1.0)$

0.05

$p=0.17$

$p=0.16$

$0.9(0.4-1.8) ;$ $0.68^{*}$

$47(89 \%)$

$6(11 \%)$

$0.8(0.3-2.2)$ 0.63

$11(20 \%)$

$32(58 \%) \quad 1.8(0.8-4.3)$

0.16

$1.3(0.5-3.6) ;$

0.57

$17(31 \%)$

$37(69 \%) \quad 0.9(0.4-1.9) ;$

0.79

$11(20 \%)$

$0.5(0.2-1.4)$

0.19

$1.4(0.5-3.9)$

$0.50^{*}$

$16(49 \%)$

$3(8 \%)$

1

$1.9(0.4-11.8) ;$ $0.53^{*}$

$\mathrm{TV}$, television, sdNVP, single dose nevirapine; VL, viral load; ART, antiretroviral therapy.

* 2-sided Fisher's exact test. 
Table 2 Characteristics at 24 weeks post-antiretroviral therapy (ART) initiation and incomplete adherence (ever with pill count $<95 \%$ past 24 weeks)

\begin{tabular}{|c|c|c|c|}
\hline Type of exposure & $\begin{array}{l}\text { Number }(\%) \text { in group who had } \\
\text { complete adherence } \\
(\mathrm{n}=92)\end{array}$ & $\begin{array}{l}\text { Number }(\%) \text { in group who had } \\
\text { incomplete adherence } \\
(\mathrm{n}=55)\end{array}$ & $\begin{array}{l}\text { Univariable } \\
\text { odds ratio } \\
\text { (Cl 95\%) } \\
\text { p value }\end{array}$ \\
\hline \multicolumn{4}{|l|}{ Quality of life } \\
\hline Good (score 12-23) $(95 ; 65 \%)$ & $64(70 \%)$ & $31(56 \%)$ & 1 \\
\hline Intermediate (score 24-35) (45; 31\%) & $25(27 \%)$ & $20(36 \%)$ & $1.6(0.8-3.4) ; 0.18$ \\
\hline Poor (score 36-55) (6; 4\%) & $2(2 \%)$ & $4(8 \%)$ & $\begin{array}{l}4.1(0.5-47.2) ; \\
0.18^{*}\end{array}$ \\
\hline \multicolumn{4}{|c|}{$\begin{array}{l}\text { Life events since ART initiation Moved to a } \\
\text { new accommodation }\end{array}$} \\
\hline No $(113 ; 77 \%)$ & $73(80 \%)$ & $40(73 \%)$ & 1 \\
\hline Yes $(33 ; 23 \%)$ & $18(20 \%)$ & $15(27 \%)$ & $1.5(0.7-3.4) ; 0.30$ \\
\hline \multicolumn{4}{|l|}{ Lost job } \\
\hline No $(138 ; 95 \%)$ & $88(97 \%)$ & $50(91 \%)$ & 1 \\
\hline Yes $(8 ; 5 \%)$ & $3(3 \%)$ & $5(9 \%)$ & $\begin{array}{l}2.9(0.5-19.5) \\
0.15^{*}\end{array}$ \\
\hline \multicolumn{4}{|l|}{ Physically attacked } \\
\hline No $(133 ; 91 \%)$ & $83(91 \%)$ & $50(91 \%)$ & 1 \\
\hline Yes $(13 ; 9 \%)$ & $8(8 \%)$ & $5(9 \%)$ & $\begin{array}{l}1.0(0.3-3.8) \\
1.00^{*}\end{array}$ \\
\hline \multicolumn{4}{|l|}{ Treated badly because of HIV status } \\
\hline No $(129 ; 88 \%)$ & $79(87 \%)$ & $50(91 \%)$ & 1 \\
\hline Yes $(17 ; 12 \%)$ & $12(13 \%)$ & $5(9 \%)$ & $\begin{array}{l}0.7(0.2-2.2) \\
0.60^{*}\end{array}$ \\
\hline \multicolumn{4}{|l|}{ Got divorced/separated } \\
\hline No $(118 ; 80 \%)$ & $78(85 \%)$ & $40(73 \%)$ & 1 \\
\hline Yes $(29 ; 20 \%)$ & $14(15 \%)$ & $15(27 \%)$ & $2.1(0.9-4.8) ; 0.08$ \\
\hline \multicolumn{4}{|l|}{ Death of a child since ART initiation } \\
\hline No $(140 ; 96 \%)$ & $88(97 \%)$ & $52(95 \%)$ & 1 \\
\hline Yes $(6 ; 4 \%)$ & $3(3 \%)$ & $3(5 \%)$ & $\begin{array}{l}1.7(0.2-13.1) \\
0.67^{*}\end{array}$ \\
\hline \multicolumn{4}{|l|}{$\begin{array}{l}\text { Death of a family member since ART } \\
\text { initiation }\end{array}$} \\
\hline No $(114 ; 78 \%)$ & $72(79 \%)$ & $42(76 \%)$ & 1 \\
\hline Yes $(32 ; 22 \%)$ & $19(21 \%)$ & $13(24 \%)$ & $1.2(0.5-2.6) ; 0.70$ \\
\hline \multicolumn{4}{|l|}{ Cumulative number of life events } \\
\hline $0(63 ; 43 \%)$ & $42(46 \%)$ & $21(38 \%)$ & 1 \\
\hline $1(52 ; 36 \%)$ & $34(37 \%)$ & $18(33 \%)$ & $1.1(0.5-2.3) ; 0.89$ \\
\hline $2(15 ; 10 \%)$ & $7(8 \%)$ & $8(15 \%)$ & $2.3(0.7-7.3) ; 0.15$ \\
\hline$\geq 3(16 ; 11 \%)$ & $8(9 \%)$ & $8(14 \%)$ & $2.0(0.6-6.2) ; 0.22$ \\
\hline
\end{tabular}

ART, antiretroviral therapy.

* 2-sided Fisher's exact test.

significant difference in viral suppression between women exposed to sdNVP 18-36 months earlier relative to exposed women with a pregnancy within the same interval [23].

\section{Different adherence levels and viral load $>400$ copies $/ \mathrm{ml}$} at week 24

Correlations between VL $>400$ copies/ml at week 24 and adherence defined using varying thresholds are shown in Table 4a. In the group overall, there was a significant association between VL $>400$ copies/ml and ever having an adherence level $<80 \%(\mathrm{p}=0.01)$ or $<70 \%(\mathrm{p}=0.01)$. Next, we assessed the correlation between VL $>400$ copies/ml and adherence defined using varying thresholds stratifying women based on their exposure to sdNVP (Table 4b). For women who had received sdNVP, there was a significant association between VL $>400$ copies/ml at week 24 and adherence levels $<95 \%$, $<90 \%,<80 \%$, and $<70 \%$ ( $\mathrm{p} \leq 0.03$ ). For women who had not been exposed to sdNVP, there was no significant association at any threshold. 
Table 3 Associations between pre-ART characteristics and viral load $>400$ copies/ml at week 24

Type of exposure $\quad$ Number (\%) in women with $\mathrm{VL} \leq 400 \quad$ Number (\%) in women with VL $>400$

Univariable odds

ratio (Cl 95\%)

p-value

\section{Pre-ART initiation $(\mathrm{n} ; \%)$ \\ Demographics \\ Ever reported pill count level \\ $<95 \%$ over 24 weeks}
No $(92 ; 63 \%)$

Yes $(55 ; 37 \%)$

Born in South Africa

$\begin{array}{ll}\text { No }(18 ; 12 \%) & 15(11 \%) \\ \text { Yes }(128 ; 88 \%) & 120(89 \%)\end{array}$

Age (years)

$\begin{array}{ll}<30(78 ; 53 \%) & 70(51 \%) \\ \geq 30(69 ; 47 \%) & 66(49 \%)\end{array}$

Education level

$>$ grade $11(54 ; 37 \%)$

$52(39 \%)$

$\leq$ grade $11(91 ; 63 \%)$

$82(61 \%)$

Marital status

Single/divorced/widow (122; 114 (84\%) 83\%)

Married/partner (25; 17\%)

$22(16 \%)$

Socio-economic status (SES)

Employed

$\begin{array}{ll}\text { No }(101 ; 69 \%) & 95(70 \%) \\ \text { Yes }(46 ; 31 \%) & 41(30 \%)\end{array}$

Type of housing

House/flat/rented room (93;

64\%)

Shack/outbuilding $(54 ; 37 \%)$

$88(65 \%)$

$48(35 \%)$

Number of children living at home
0-1 child (89; 60\%)
$\geq 2$ children $(58 ; 40 \%)$
$80(59 \%)$
$56(41 \%)$

Lack of financial support from partner/husband

Support $(67 ; 45 \%)$
No support $(80 ; 55 \%)$

$62(45 \%)$

$74(54 \%)$

Water source inside home
No $(74 ; 50 \%)$
Yes $(73 ; 50 \%)$
$68(50 \%)$
$68(50 \%)$

Toilet at home

No $(86 ; 59 \%)$

Yes $(61 ; 41 \%)$

78 (57\%)

58 (43\%)

$22(16 \%)$

114 (84\%)

No $(23 ; 16 \%)$

Yes $(124 ; 84 \%)$

Refrigerator at home

$\begin{array}{ll}\text { No }(50 ; 34 \%) & 47(35 \%) \\ \text { Yes }(97 ; 66 \%) & 89(65 \%)\end{array}$

Radio at home

No $(28 ; 19 \%)$

Yes $(119 ; 81 \%)$
89 (65\%)

26 (19\%)

$110(81 \%)$
$5(45 \%)$

$6(55 \%)$

$2.1(0.5-9.3) ; 0.33^{*}$

$3(27 \%)$

$8(73 \%)$

$0.3(0.1-2.2) ; 0.14^{*}$

$8(72.7 \%)$

1

$3(27.3 \%)$

$0.4(0.1-1.7) ; 0.22^{*}$

$2(18 \%)$

1

$9(82 \%)$

$2.9(0.6-28.0) ; 0.21^{*}$

$8(73 \%)$

$3(27 \%)$

$1.9(0.3-8.9) ; 0.40^{*}$

$6(55 \%)$

$5(45 \%)$

$1.9(0.4-8.0) ; 0.32^{*}$

$5(45 \%)$

1

$6(55 \%)$

$2.2(0.5-9.6) ; 0.21^{*}$

$9(82 \%)$

$2(18 \%)$

1

$0.3(0-1.6) ; 0.20^{*}$

$5(45 \%)$

1

$1.00(0.2-4.1) ; 1.00^{*}$

$6(55 \%)$

$5(45 \%)$

1

$0.8(0.2-3.5) ; 1.00^{*}$

8 (73\%)

$3(27 \%)$

1

$0.5(0.1-2.2) ; 0.36^{*}$

1 (9\%)

10 (91\%)

$1.9(0.2-87.5) ; 1.0^{*}$

$3(27 \%)$

$1.4(0.3-8.6) ; 0.75^{*}$

$2(18 \%)$

1

$1.1(0.2-10.7) ; 1.00^{*}$ 
Table 3 Associations between pre-ART characteristics and viral load $>400$ copies/ml at week 24 (Continued)

\section{TV at home}

$\begin{array}{ll}\text { No }(27 ; 18 \%) & 25(18 \%) \\ \text { Yes }(120 ; 82 \%) & 111(82 \%)\end{array}$

Landline telephone
No $(131 ; 89 \%)$
$121(89 \%)$
Yes $(16 ; 11 \%)$
$15(11 \%)$

Pre-treatment clinical

characteristics

Exposed to sdNVP

$\begin{array}{ll}\text { No }(57 ; 39 \%) & 51(38 \%) \\ \text { Yes }(90 ; 61 \%) & 85(62 \%)\end{array}$

VL level (copies/ml) pre-ART

Median (range)

$85(62 \%)$

110,000

$(760->750,000)$

CD4 cell count - pre-ART

Median (range)

$164(2-430)$

Quality of life (pre-ART)

Good (score 12-23) (38; 26\%) 34 (25\%)

Intermediate (score 24-35) (75; 70 (51\%)

51\%)

Poor (score 36-55) (34; 23\%)

$32(24 \%)$

Disclosure and social support

Disclosed HIV status
No $(44 ; 30 \%)$
Yes (102; 70\%)
$42(31 \%)$
94 (69\%)

Treatment buddy

$$
\begin{array}{ll}
\text { No }(22 ; 15 \%) & 21(15 \%) \\
\text { Yes }(125 ; 85 \%) & 115(85 \%)
\end{array}
$$

Another adult at home, living with HIV
No $(46 ; 57 \%)$
Yes $(35 ; 43 \%)$
$43(57 \%)$
$32(43 \%)$

Another child at home, living with HIV

$$
\begin{array}{ll}
\text { No }(12 ; 12 \%) & 12(13 \%) \\
\text { Yes }(87 ; 88 \%) & 79(87 \%)
\end{array}
$$

At week 24

Quality of life (post-ART)

Good (score 12-23) (95; 65\%) 88 (65\%)

Intermediate (score 24-35) (45; 42 (31\%)

31\%)

Poor (score 36-55) (6; 4\%) $5(4 \%)$

\section{Moved to a new}

accommodation

$$
\begin{array}{ll}
\text { No }(113 ; 77 \%) & 105(78 \%) \\
\text { Yes }(33 ; 23 \%) & 30(22 \%)
\end{array}
$$

Lost job

$\begin{array}{ll}\text { No }(138 ; 95 \%) & 128(95 \%) \\ \text { Yes }(8 ; 5 \%) & 7(5 \%)\end{array}$

Physically attacked
No (133; 91\%)
$122(90 \%)$
Yes $(13 ; 9 \%)$
$13(10 \%)$

\section{$2(18 \%)$}

$9(82 \%)$

$10(91 \%)$

$1(9 \%)$

$6(55 \%)$

$0.5(0.1-2.1) ; 0.34^{*}$

92,300

$(6,600-359,000)$

$p=0.34$

$191(7-355)$

$p=0.91$

$4(36 \%)$

5 (46\%)

$2(18 \%)$

$0.6(0.1-3.3) ; 0.48^{*}$

0.5 (0-4.0); $0.68^{*}$

$2(20 \%)$

1

$8(80 \%)$

$1.8(0.3-17.9) ; 0.72^{*}$

1 (9\%)

$10(91 \%)$

1

$1.8(0.2-93.0) ; 1.00^{*}$

$3(50 \%)$

$3(50 \%)$

1

$1.3(0.2-10.7) ; 1.00^{*}$

0

$8(100 \%)$

NA; 0.59*

$7(64 \%)$

1

$3(27 \%)$

$0.9(0.1-4.2) ; 1.00^{*}$

$1(9 \%)$

$2.5(0-27.4) ; 0.40^{*}$

8 (73\%)

$3(27 \%)$

1

$1.3(0.2-5.9) ; 0.71^{*}$

$10(91 \%)$

$1(9 \%)$

1

$1.8(0-16.7) ; 0.47^{*}$

11 (100\%)

0

1

NA 
Table 3 Associations between pre-ART characteristics and viral load $>\mathbf{4 0 0}$ copies/ml at week 24 (Continued)

\begin{tabular}{|c|c|c|c|}
\hline \multicolumn{4}{|c|}{$\begin{array}{l}\text { Treated badly because of HIV } \\
\text { status }\end{array}$} \\
\hline No $(129 ; 88 \%)$ & $120(89 \%)$ & $9(82 \%)$ & 1 \\
\hline Yes $(17 ; 12 \%)$ & $15(11 \%)$ & $2(18 \%)$ & $1.8(0.2-9.8) ; 0.62^{*}$ \\
\hline \multicolumn{4}{|c|}{ Got divorced/separated } \\
\hline No $(118 ; 80 \%)$ & $109(80 \%)$ & $9(82 \%)$ & 1 \\
\hline Yes $(29 ; 20 \%)$ & $27(20 \%)$ & $2(18 \%)$ & $0.9(0.1-4.7) ; 1.00^{*}$ \\
\hline \multicolumn{4}{|c|}{ A child died since ART initiation } \\
\hline No $(140 ; 96 \%)$ & $130(96 \%)$ & $10(91 \%)$ & 1 \\
\hline Yes $(6 ; 4 \%)$ & $5(4 \%)$ & $1(9 \%)$ & $2.6(0.1-26.6) ; 0.38^{*}$ \\
\hline \multicolumn{4}{|c|}{$\begin{array}{l}\text { Any family member died since } \\
\text { ART initiation }\end{array}$} \\
\hline No $(114 ; 78 \%)$ & $108(80 \%)$ & $6(55 \%)$ & 1 \\
\hline Yes $(32 ; 22 \%)$ & $27(20 \%)$ & $5(45 \%)$ & $3.3(0.7-14.0) ; 0.06^{*}$ \\
\hline \multicolumn{4}{|c|}{$\begin{array}{l}\text { Cumulative number of life } \\
\text { events }\end{array}$} \\
\hline $0(63 ; 43 \%)$ & $59(44 \%)$ & $4(36 \%)$ & 1 \\
\hline $1(52 ; 36 \%)$ & $48(36 \%)$ & $4(36 \%)$ & $1.2(0.2-6.9) ; 1.00^{*}$ \\
\hline $2(15 ; 10 \%)$ & $14(10 \%)$ & $1(9 \%)$ & $1.1(0-11.8) ; 1.00^{*}$ \\
\hline$\geq 3(16 ; 11 \%)$ & $14(10 \%)$ & $2(19 \%)$ & $2.1(0.2-16.3) ; 0.59^{*}$ \\
\hline
\end{tabular}

TV, television, sdNVP, single dose nevirapine; VL, viral load; ART, antiretroviral therapy.

* 2-sided Fisher's exact test.

\section{Barriers to adherence}

Potential barriers to adherence endorsed by study participants are shown in Figure 1. The women reported three main reasons for missing their medication: being away from home, being busy with other things and simply forgetting. The most common reason for missing pills, viz being away from home, varied in importance over the first 24 weeks. Other reasons for missing daily medications remained similar over weeks 2-20 diminishing in importance by week 24 .

\section{Discussion}

In this cohort of women starting ART in Johannesburg and who received extensive adherence support, we explored the association between socio-economic characteristics, adherence and virologic failure during the first 24 weeks of treatment. There was a high level of adherence despite the social and economic challenges faced by these participants, many of whom were single mothers living in disadvantaged economic circumstances. These observations are consistent with other studies from SSA [28-31] and reflect patients' commitment to HIV care in South Africa [28].

Reduced adherence, measured as a percent of doses missed in a given time period, has been associated with virologic failure. The relationship between adherence and virologic failure appears to be affected by the classes of drugs used and may differ between early and later treatment $[32,33]$. In our data, it appeared that previous use of sdNVP may affect the relationship between adherence and virologic failure of an NNRTI-containing regimen. In the total study population, less than $80 \%$ adherence was significantly associated with virologic failure. However, when women were stratified by exposure to sdNVP, the effect of adherence on virologic failure was strongest among the sdNVP exposed women, where anything less than 95\% adherence was significantly associated with increased virologic failure. This might be due to the selection and persistence of NVP resistance mutations, albeit as minority species undetected in consensus sequence [34]. In addition, the higher the level of adherence to NNRTI-based therapy, the lower the risk of drug resistance and virologic failure [18,32]. This contrasts with the unexposed women among whom there was no association between reduced levels of adherence and virologic failure. This difference may be a result of the relatively small number of unexposed women $(n=57)$ and should be confirmed in larger prospective studies. Nevertheless, our findings raise the question of whether drug resistance selected by sdNVP may be partially overcome with complete adherence.

The primary study was designed to assess the effect of sdNVP on virologic response to therapy. Although exposure to sdNVP in the prior 18-36 months was not associated with a reduced likelihood of achieving and sustaining viral suppression, women with minority K103N mutations before treatment had a reduced durability of virologic suppression [23] as observed in other studies $[20,22,35,36]$. Some studies, however, have reported poor virologic response to NNRTI-based 
Table 4 Correlation between different adherence levels and VL >400 copies/ml at week 24: a) among all women and b) among women exposed vs. not exposed to sdNVP separately

\begin{tabular}{|c|c|c|c|}
\hline \multirow{2}{*}{$\begin{array}{l}\text { a) } \\
\text { Percent adherence during any of the visits } 2-24 \text { weeks }\end{array}$} & \multicolumn{2}{|c|}{ All women } & \multirow[b]{2}{*}{$\begin{array}{l}\text { Univariable odds ratio } \\
\text { (Cl 95\%); } p \text { value }\end{array}$} \\
\hline & $\leq 400$ copies $/ \mathrm{ml}$ & $>400$ copies $/ \mathrm{ml}$ & \\
\hline $100 \%(n=28)$ & $27(20 \%)$ & $1(9 \%)$ & 1 \\
\hline Ever $<100 \%(n=119)$ & 109 (80\%) & 10 (91\%) & $2.5(0.3-111.4) ; 0.69^{*}$ \\
\hline$>95 \%(n=92)$ & 87 (64\%) & $5(45 \%)$ & 1 \\
\hline Ever $<95 \%(n=55)$ & $49(36 \%)$ & $6(55 \%)$ & $2.1(0.5-9.3) ; 0.33^{*}$ \\
\hline$>90 \%(n=105)$ & $99(73 \%)$ & $6(55 \%)$ & 1 \\
\hline Ever $<90 \%(n=42)$ & $37(27 \%)$ & $5(45 \%)$ & $2.2(0.5-9.3) ; 0.30^{*}$ \\
\hline$>80 \%(n=122)$ & $116(85 \%)$ & $6(55 \%)$ & 1 \\
\hline Ever $<80 \%(n=25)$ & $20(15 \%)$ & $5(45 \%)$ & $4.8(1.0-20.8) ; 0.02^{*}$ \\
\hline$>70 \%(n=137)$ & $129(95 \%)$ & $8(73 \%)$ & 1 \\
\hline Ever $<70 \%(n=10)$ & 7 (5\%) & $3(27 \%)$ & $6.9(1.0-37.7) ; 0.03^{*}$ \\
\hline b) & \multicolumn{2}{|c|}{ Exposed to sdNVP } & \\
\hline Percent adherence during any of the visits $2-24$ weeks & $\leq 400$ copies $/ \mathrm{ml}$ & $>400$ copies $/ \mathrm{ml}$ & $\begin{array}{l}\text { Univariable odds ratio } \\
\text { (Cl 95\%); } p \text { value }\end{array}$ \\
\hline $100 \%(n=18)$ & $18(21 \%)$ & 0 & 1 \\
\hline Ever $<100 \%(n=72)$ & $67(79 \%)$ & $5(100 \%)$ & NA: $0.58^{*}$ \\
\hline$>95 \%(n=62)$ & $61(72 \%)$ & $1(20 \%)$ & 1 \\
\hline Ever $<95 \%(n=28)$ & $24(28 \%)$ & $4(80 \%)$ & $10.2(0.9-508.5) ; 0.03^{*}$ \\
\hline$>90 \%(n=69)$ & $68(80 \%)$ & $1(20 \%)$ & 1 \\
\hline Ever $<90 \%(n=21)$ & $17(20 \%)$ & $4(80 \%)$ & 16 (1.4-798.9); 0.01* \\
\hline$>80 \%(n=75)$ & $74(87 \%)$ & $1(20 \%)$ & 1 \\
\hline Ever $<80 \%(n=15)$ & $11(13 \%)$ & $4(80 \%)$ & $26.9(2.2-1341.7) ;<0.01 *$ \\
\hline$>70 \%(n=83)$ & $80(94 \%)$ & $3(60 \%)$ & 1 \\
\hline \multirow[t]{3}{*}{ Ever $<70 \%(n=7)$} & $5(6 \%)$ & $2(40 \%)$ & $10.7(0.7-112.3) ; 0.01^{*}$ \\
\hline & \multicolumn{2}{|c|}{ Not exposed to sdNVP } & \\
\hline & $\leq 400$ copies $/ \mathrm{ml}$ & $>400$ copies $/ \mathrm{ml}$ & $\begin{array}{l}\text { Univariable odds ratio } \\
\text { (Cl 95\%); p value }\end{array}$ \\
\hline $100 \%(n=10)$ & $9(18 \%)$ & $1(17 \%)$ & 1 \\
\hline Ever $<100 \%(n=47)$ & $42(82 \%)$ & $5(83 \%)$ & $1.1(0.1-56.2) ; 1.00^{*}$ \\
\hline$>95 \%(n=30)$ & $26(51 \%)$ & $4(67 \%)$ & 1 \\
\hline Ever $<95 \%(n=27)$ & $25(49 \%)$ & $2(33 \%)$ & $0.5(0-4.1) ; 0.67^{*}$ \\
\hline$>90 \%(n=36)$ & $31(61 \%)$ & $5(83 \%)$ & 1 \\
\hline Ever $<90 \%(n=21)$ & 20 (39\%) & $1(17 \%)$ & $0.3(0-3.1) ; 0.40^{*}$ \\
\hline$>80 \%(n=47)$ & $42(82 \%)$ & $5(83 \%)$ & 1 \\
\hline Ever $<80 \%(n=10)$ & $9(18 \%)$ & $1(17 \%)$ & $0.9(0-10.0) ; 1.00^{*}$ \\
\hline$>70 \%(n=54)$ & $49(96 \%)$ & $5(83 \%)$ & 1 \\
\hline Ever <70\% $(n=3)$ & $2(4 \%)$ & $1(17 \%)$ & $4.9(0.1-106.0) ; 0.29^{*}$ \\
\hline
\end{tabular}

sdNVP, single dose nevirapine; VL, viral load.

* 2-sided Fisher's exact test.

therapy even if exposure is quite distant [37]. These differences between studies may, in part be explained by different levels of adherence. If the effects of incomplete adherence on virologic suppression depend on past drug exposure, as suggested by our data, studies with less attention to sustaining high levels of adherence may detect decreased efficacy of ART among those with past exposure to sdNVP. Since current guidelines recommend that pregnant women with $<350 \mathrm{CD} 4+$ cells $/ \mathrm{mm}^{3}$ receive $A R T$, rather than $\mathrm{SdNVP}$, there is less basis to be concerned about the impact of sdNVP on later treatment outcomes [38]. Adherence level to ART is found to be high among HIV infected pregnant women [39] compared to non-pregnant women $[40,41]$. However, ART is a life-time treatment and sustaining high adherence level should remain the ultimate goal.

The women who had participated in the pMTCT program were more likely to be adherent to ART in univariable analysis although this association did not remain after adjustment for social characteristics. 


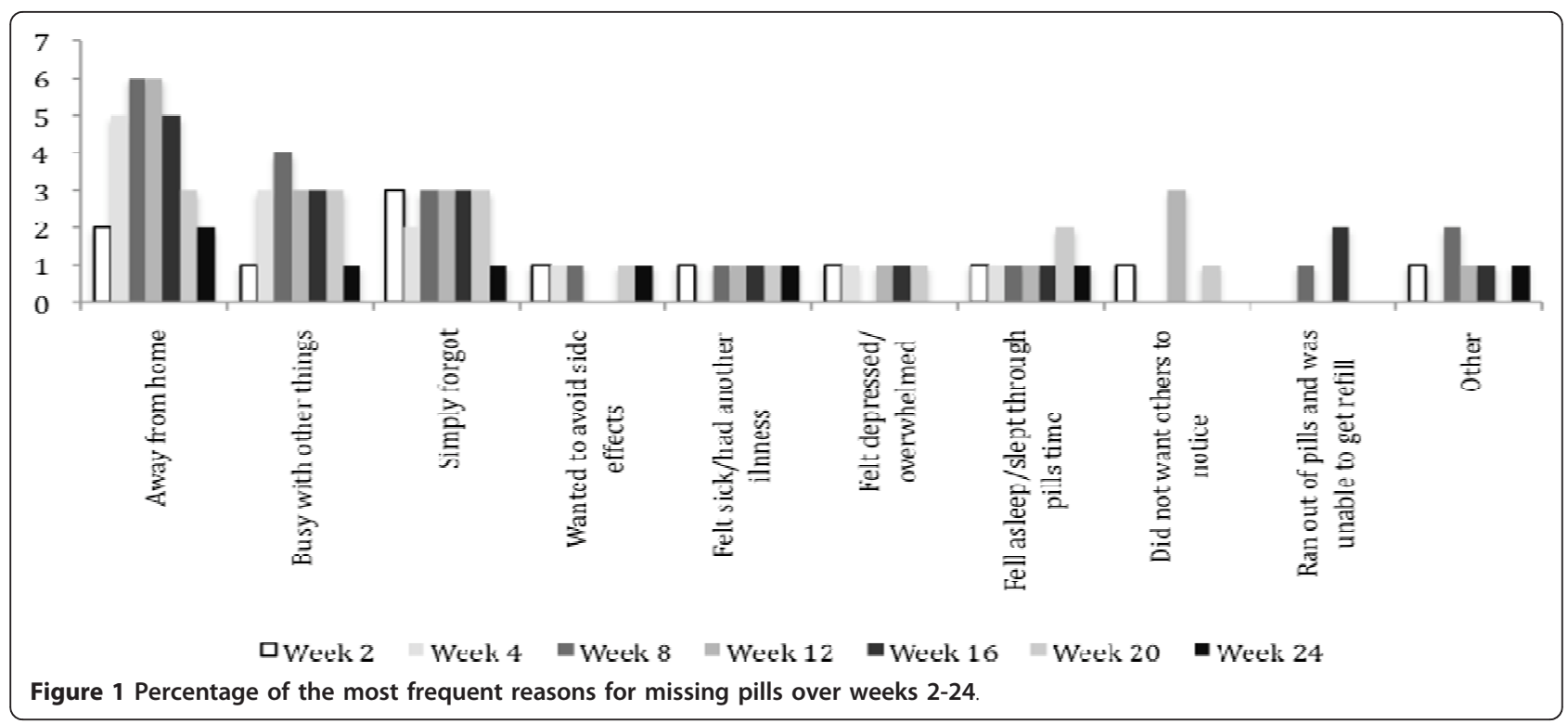

Increased treatment preparation in pMTCT programs may provide women with information about ART and adherence as shown in a study in Uganda [42]. Another study [43] at a rural district hospital in South Africa demonstrated that women provided with partner support and complete pMTCT information were more likely to take sdNVP. Thus, programs that provide sdNVP for $\mathrm{pMTCT}$ and provide women with increased treatment education, information and support are likely to achieve better adherence on ART. Here, women, with less education were at higher risk for incomplete adherence. Other studies of women [44] and of men and women [45] found independent associations between education, health literacy and adherence to ART. An additional concern is women's understanding of their children's treatment needs, where poor education may pose an additional risk for inadequate administration of ART to their infected children [46].

In Johannesburg, less education, living in an informal setting and providing care for at least two children with uncertain partner support, were each associated with reduced adherence and an increased need for support [47]. Ware and co-workers [48] found, in a large ethnographic study in Nigeria, Tanzania and Uganda, that social support enhances adherence. Also Merenstein and co-workers [7] found that having the sole responsibility for a child reduces adherence among women in the United States. Lack of potable water at home as reported by Ellis and co-workers in Nairobi was associated with incomplete adherence among both adults and children [49]. In this study, we observed that patients with low socio-economic status, assessed by living in an informal dwelling, the absence of a TV or lack of potable water at home were each associated with incomplete adherence although these did not retain significance in the final model, possibly because of the small sample size. The three main reasons for missing pills were being away from home, being busy with other things and simply forgetting, which are consistent with the findings of a review by Mills and co-workers [50].

There are several limitations to the generalizability of this study. Postpartum women are a vulnerable population who may have particular difficulty in obtaining access to ART due to financial challenges as described by Rosen and co-workers [51]. Here we followed a relatively small number of women for only six months and did not monitor changes in socioeconomic status, relationships, employment or depression over time. Rather, we relied on baseline responses and recall at six months. Reimbursement for transport costs, estimated to be $\sim 25 \%$ of the patients' cost of ART [51], may have improved adherence to treatment [52]. In addition, this was a clinical research study in which attention and resources were devoted to reinforcing adherence [53], so it is likely that incomplete adherence may be more prevalent in a routine HIV care setting. Further limitations include focus only on a single measure of adherence and uncertain validity of some of the co-factors investigated, including QOL. Adherence to ART is a dynamic process influenced by psychosocial and socio-economic factors that need further investigation [54].

\section{Conclusions}

In conclusion, women receiving an NNRTI-based regimen may achieve virologic suppression with $>80 \%$ adherence. However, higher levels of adherence may be necessary among women who have been exposed to 
sdNVP. Women starting on ART with less education and lower socio-economic level were also at a higher risk of incomplete adherence to ART over the first six months of treatment and special adherence counseling and interventions may be needed to ensure sufficient support for long-term sustainability of treatment response among these women.

\section{Acknowledgements}

We thank the Karolinska Institutet Faculty Funds (KID), the Swedish International Development Agency (Sida) and the National Institute for Communicable Diseases (NICD) for supporting this study. Funding for the original study was via the National Institutes of Child Health and Human Development (HD 47177), and Secure the Future Foundation.

\section{Author details}

'Division of Global Health (IHCAR), Karolinska Institutet, Stockholm, Sweden. ${ }^{2}$ AIDS Virus Research Unit, National Institute for Communicable Diseases (NICD), Johannesburg, South Africa. ${ }^{3}$ Empilweni Services and Research Unit, Rahima Moosa Mother and Child Hospital, Faculty of Health Sciences, University of the Witwatersrand, Johannesburg, South Africa. ${ }^{4}$ International Center for AIDS Programs, Mailman School of Public Health, Columbia University, New York, USA. ${ }^{5}$ Nordic School of Public Health (NHV), Gothenburg, Sweden. 'Division of Infectious Diseases, Stanford University, California, USA. ${ }^{7}$ Gertrude H. Sergievsky Center, College of Physicians and Surgeons, and Department of Epidemiology, Mailman School of Public Health, Columbia University, New York, NY, USA.

\section{Authors' contributions}

$E J A, A C, L M$ and LK participated in study design, ethical clearance and data collection. ZEK, AME, MP, DK, LM and LK participated in data analysis. All authors read and approved the final manuscript.

\section{Competing interests}

The authors declare that they have no competing interests.

Received: 26 November 2010 Accepted: 8 February 2011 Published: 8 February 2011

\section{References}

1. World Health Organization (WHO), UNAIDS, UNICEF: Towards universal access - Scaling up priority HIV/AIDS interventions in the health sector (progress report 2009). Geneve. 2009.

2. World Health Organization (WHO): AIDS epidemic update. Geneve. 2009

3. Dembele M, Saleri N, Carvalho AC, Saouadogo T, Hien AD, Zabsonre I, Koala ST, Simpore J, Matteelli A: Incidence of tuberculosis after HAART initiation in a cohort of HIV-positive patients in Burkina Faso. Int I Tuberc Lung Dis 2010, 14:318-323.

4. Muula AS, Ngulube TJ, Siziya S, Makupe CM, Umar E, Prozesky HW, Wiysonge CS, Mataya RH: Gender distribution of adult patients on highly active antiretroviral therapy (HAART) in Southern Africa: a systematic review. BMC Public Health 2007, 7:63.

5. Cornell M, Myer L, Kaplan R, Bekker L, Wood R: The impact of gender and income on survival and retention in a South African antiretroviral therapy programme. Trop Med Int Health 2009, 14:722-731.

6. Kempf MC, Pisu M, Dumcheva A, Westfall AO, Md JM, Saag MS: Gender differences in discontinuation of antiretroviral treatment regimens. $J$ Acquir Immune Defic Syndr 2009, 52:336-341.

7. Merenstein D, Schneider M, Cox C, Schwartz R, Weber K, Robison E, Gandhi M, Richardson J, MW P: Association of child care burden and household composition with adherence to highly active antiretroviral therapy in the women's interagency HIV study. AIDS Patient Care and STDs 2009, 23:289-296.

8. Vyavaharkar M, Moneyham L, Tavakoli A, Phillips KD, Murdaugh C, Jackson K, Meding G: Social support, coping, and medication adherence among HIV-positive women with depression living in rural areas of the southeastern United States. AIDS Patient Care STDS 2007, 21:667-680.
9. Murray LK, Semrau K, McCurley E, Thea DM, Scott N, Mwiya M, Kankasa C, Bass J, Bolton P: Barriers to acceptance and adherence of antiretroviral therapy in urban Zambian women: a qualitative study. AIDS Care 2009, 21:78-86.

10. Shuter J: Forgiveness of non-adherence to HIV-1 antiretroviral therapy. Journal of Antimicrobial Chemotherapy 2008, 61(4):769-73.

11. Paterson DL, Swindells S, Mohr J, Brester M, Vergis EN, Squier C, Wagener MM, Singh N: Adherence to protease inhibitor therapy and outcomes in patients with HIV infection. Ann Intern Med 2000, $136: 21-30$.

12. Nachega JB, Hislop M, Dowdy DW, Chaisson RE, Regensberg L, Maartens G: Adherence to nonnucleoside reverse transcriptase inhibitor-based HIV therapy and virologic outcomes. Ann Intern Med 2007, 146:564-574.

13. Shuter J, Sarlo J, Kanmaz T, Rode R, Zingman B: HIV-infected patients receiving lopinavir/ritonavir-based antiretroviral therapy achieve high rates of virologic suppression despite adherence rates less than $95 \%$. $J$ Acquir Immune Defic Syndr 2007, 45:4-8.

14. Martin M, Del Cacho E, Codina C, Tuset M, De Lazzari E, Mallolas J, Miro JM, Gatell JM, Ribas J: Relationship between adherence level, type of the antiretroviral regimen, and plasma HIV type 1 RNA viral load: A prospective cohort study. AIDS Res Hum Retrovirus 2008, 24:1263-1268.

15. Bangsberg DR: Less than $95 \%$ adherence to nonnucleoside reversetranscriptase inhibitor therapy can lead to viral suppression. Clin Infect Dis 2006, 43:939-941.

16. Lima VD, Harrigan R, Murray M, Moore DM, Wood E, Hogg RS, Montaner JS: Differential impact of adherence on long-term treatment response among naive HIV-infected individuals. AIDS 2008, 22:2371-2380.

17. Low-Beer S, Yip B, O'Shaughnessy M, Hogg R, Montaner J: Adherence to triple therapy and viral load response. J Acquir Immune Defic Syndr 2000, 23:360-361.

18. Bangsberg DR, Moss AR, Deeks SG: Paradoxes of adherence and drug resistance to HIV antiretroviral therapy. J Antimicrob Chemother 2004, 53:696-699.

19. Ford N, Darder M, Spelman T, Maclean E, Mills E, Boulle A: Early adherence to antiretroviral medication as a predictor of long-term HIV virological suppression: five-year follow up of an observational cohort. PLoS One 2010, 5:e10460.

20. Lockman S, Shapiro RL, Smeaton LM, Wester C, Thior I, Stevens L, Chand F, Makhema J, Moffat C, Asmelash A, et al: Response to antiretroviral therapy after a single, peripartum dose of nevirapine. N Engl J Med 2007, 356:135-147.

21. Lallemant M, Jourdain G, Le Coeur S, Mary JY, Ngo-Giang-Huong N, Koetsawang S, Kanshana S, McIntosh K, Thaineua V: Single-dose perinatal nevirapine plus standard zidovudine to prevent mother-to-child transmission of HIV-1 in Thailand. N Engl J Med 2004, 351:217-228.

22. Chintu N, Giganti MJ, Putta NB, Sinkala M, Sadoki E, Stringer EM, Stringer JS, Chi BH: Peripartum nevirapine exposure and subsequent clinical outcomes among HIV-infected women receiving antiretroviral therapy for at least 12 months. Trop Med Int Health 2010, 15:842-847.

23. Coovadia A, Hunt G, Abrams EJ, Sherman G, Meyers T, Barry G, Malan E, Marais B, Stehlau R, Ledwaba J, et al: Persistent minority K103N mutations among women exposed to single-dose nevirapine and virologic response to Nonnucleoside Reverse-Transcriptase Inhibitor-based therapy. Clin Infect Dis 2009, 48:462-472.

24. Bing E, Hays R, Jacobson L, Chen B, Gange S, Kass N, Chmiel J, Zucconi S: Health-related quality of life among people with HIV disease: results from the multicenter AIDS cohort study. Qual Life Res 2000, 9:55-63.

25. Ware J, Snow K, Kosisnki M, Gandek B: SF-36 health survey manual and interpretation guide. Boston: The Health Institute; 1993.

26. ACTG Outcomes Committee: ACTG QOL 601-602 (QOL 601-2) Health Survey Manual. 1999.

27. Grossberg R, Gross R: Use of pharmacy refill data as a measure of antiretroviral adherence. Current HIV/AIDS Reports 2007, 4:187-191.

28. Nachega JB, Stein DM, Lehman DA, Hlatshwayo D, Mothopeng R, Chaisson RE, Karstaedt AS: Adherence to antiretroviral therapy in HIV-infected adults in Soweto, South Africa. AIDS Res Hum Retrovirus 2004, 20:1053-1056.

29. Bisson GP, Rowh A, Weinstein R, Gaolathe T, Frank I, Gross R: Antiretroviral failure despite high levels of adherence: Discordant adherence-response relationship in Botswana. J Acquir Immune Defic Syndr 2008, 49:107-110

30. Mills EJ, Nachega JB, Buchan I, Orbinski J, Attaran A, Singh S, Rachlis B, Wu P, Cooper C, Thanane L, et al: Adherence to antiretroviral therapy in Sub-Saharan Africa and North America. JAMA 2006, 296:679-690. 
31. El-Khatib Z, Ekstrom AM, Ledwaba J, Mohapi L, Laher F, Karstaedt A Charalambous S, Petzold M, Katzenstein D, Morris L: Viremia and drug resistance among HIV-1 patients on antiretroviral treatment: a crosssectional study in Soweto, South Africa. AIDS 2010, 24:1679-1687.

32. Bangsberg DR, Acosta EP, Gupta R, Guzman D, Riley ED, Harrigan PR, Parkin N, Deeks SG: Adherence-resistance relationships for protease and non-nucleoside reverse transcriptase inhibitors explained by virological fitness. AIDS 2006, 20:223-231.

33. Nachega JB, Hislop M, Nguyen H, Dowdy DW, Chaisson RE, Regensberg L, Cotton M, Maartens G: Antiretroviral therapy adherence, virologic and immunologic outcomes in adolescents compared with adults in Southern Africa. J Acquir Immune Defic Syndr 2009, 51:65-71.

34. Gardner EM, Burmana WJ, Steiner JF, Andersond PL, Bangsberg DR: Antiretroviral medication adherence and the development of classspecific antiretroviral resistance. AIDS 2009, 23.

35. Jourdain G, Ngo-Giang-Huong N, Le Coeur S, Bowonwatanuwong C, Kantipong P, Leechanachai P, Ariyadej S, Leenasirimakul P, Hammer S, Lallemant M, Perinatal HIV Prevention Trial Group: Intrapartum exposure to nevirapine and subsequent maternal responses to nevirapine-based antiretroviral therapy. N Engl J Med 2007, 356:135-147.

36. Coffie PA, Tonwe-Gold B, Tanon AK, Amani-Bosse C, Bedikou G, Abrams EJ, Dabis F, Ekouevi DK: Incidence and risk factors of severe adverse events with nevirapine-based antiretroviral therapy in HIV-infected women. MTCT-Plus program, Abidjan, Cote d'Ivoire. BMC Infect Dis 2010, 10:188.

37. Datay MI, Boulle A, Mant D, Yudkin P: Associations with virologic treatment failure in adults on antiretroviral therapy in South Africa. $J$ Acquir Immune Defic Syndr 2010, 54:489-495.

38. World Health Organization (WHO): Antiretroviral therapy for HIV infection in adults and adolescents. Geneva. 2010

39. Lockman S, Hughes MD, Mclntyre J, Zheng Y, Chipato T, Conradie F, Sawe F, Asmelash A, Hosseinipour MC, Mohapi L, et al: Antiretroviral therapies in women after single-dose nevirapine exposure. N Engl J Med 2010, 363:1499-1509.

40. Vaz M, Barros S, Palacios R, Senise J, Lunardi L, Amed A, Castelo A: HIVinfected pregnant women have greater adherence with antiretroviral drugs than non-pregnant women. Int J STD AIDS 2007, 18:28-32.

41. Zorrilla C, Santiago L, Knubson D, Liberatore K, Estronza G, Colón O, Acevedo M: Greater adherence to highly active antiretroviral therapy (HAART) between pregnant versus non-pregnant women living with HIV. Cell Mol Biol (Noisy-le-grand) 2003, 49:1187-1192.

42. Nassali M, Nakanjako D, Kyabayinze D, Beyeza J, Okoth A, Mutyaba T: Access to HIV/AIDS care for mothers and children in sub-Saharan Africa: adherence to the postnatal PMTCT program. AIDS Care 2009, 21:1124-1131.

43. Peltzer K, Mlambo M, Phaswana-Mafuya N, Ladzani R: Determinants of adherence to a single-dose nevirapine regimen for the prevention of mother-to-child HIV transmission in Gert Sibande district in South Africa. Acta Paediatr 2010, 99:699-704.

44. Kalichman SC, Ramachandran B, Catz S: Adherence to combination antiretroviral therapies in HIV patients of low health literacy. J Gen Intern Med 1999, 14:267-273.

45. Golin CE, Liu H, Hays RD, Miller LG, Beck CK, Ickovics J, Kaplan AH, Wenger NS: A prospective study of predictors of adherence to combination antiretroviral medication. J Gen Intern Med 2002, 17:756-765.

46. Paranthaman K, Kumarasamy N, Bella D, Webster P: Factors influencing adherence to anti-retroviral treatment in children with human immunodeficiency virus in South India-a qualitative study. AIDS Care 2009, 21:1025-1031.

47. van Driel M: The social grants and black women in South Africa: a case study of Bophelong township in Gauteng (PhD thesis). The University of Witwatersrand, Social sciences; 2009.

48. Ware NC, Idoko J, Kaaya S, Biraro IA, Wyatt MA, Agbaji O, Chalamilla G, Bangsberg DR: Explaining adherence success in sub-Saharan Africa: An ethnographic study. PLoS Med 2009, 6:e11.

49. Ellis AE, Gogel RP, Roman BR, Watson JB, Indyk D, Rosenberg G: The STARK study: a cross-sectional study of adherence to short-term drug regiments in urban Kenya. Soc Work Health Care 2006, 42:237-250.

50. Mills EJ, Nachega JB, Bangsberg DR, Singh S, Rachlis B, Wu P, Wilson K, Buchan L, Gill CJ, Cooper C: Adherence to HAART: A systematic review of developed and developing nation patient-reported barriers and facilitators. PLoS Med 2006, 3:1-26.
51. Rosen S, Ketlhapile M, Sanne I, Desilva MB: Characteristics of patients accessing care and treatment for HIV/AIDS at public and nongovernmental sites in South Africa. $J$ Int Assoc Physicians AIDS Care (Chic III) 2008, 7:200-207.

52. Volpp K, Pauly M, Loewenstein G, Bangsberg DR: P4P4P: An agenda for research on pay-for-performance for patients. Health Affair 2009, 28:206-214.

53. Rosen S, Ketlhapile M, Sanne I, DeSilva MB: Cost to patients of obtaining treatment for HIV/AIDS in South Africa. S Afr Med J 2007, 97:524-529.

54. Amberbir A, Woldemichael K, Getachew S, Girma B, Deribe K: Predictors of adherence to antiretroviral therapy among HIV-infected persons: a prospective study in Southwest Ethiopia. BMC Health Serv Res 2008, 8:265.

\section{Pre-publication history}

The pre-publication history for this paper can be accessed here:

http://www.biomedcentral.com/1471-2458/11/88/prepub

doi:10.1186/1471-2458-11-88

Cite this article as: El-Khatib et al:: Adherence and virologic suppression during the first 24 weeks on antiretroviral therapy among women in Johannesburg, South Africa - a prospective cohort study. BMC Public Health 2011 11:88.

\section{Submit your next manuscript to BioMed Central and take full advantage of:}

- Convenient online submission

- Thorough peer review

- No space constraints or color figure charges

- Immediate publication on acceptance

- Inclusion in PubMed, CAS, Scopus and Google Scholar

- Research which is freely available for redistribution

Submit your manuscript at www.biomedcentral.com/submit
Biomed Central 